\title{
Hábitos de lectura y afinidad tecnológica de los estudiantes universitarios: estudio comparativo de cinco universidades de habla hispana
}

(Reading Habits and Technological Affinity of University Students: A Comparative Study of Five Spanish-speaking Universities)

LILIAN IVETTHE SALADO RODRÍGUEZ ${ }^{\mathbb{D}}$, Universidad Estatal de Sonora, Sonora, México Alberto RAMÍREZ-MARTINELL ${ }^{\mathbb{D}}$, Universidad Veracruqana, Veracruq, México REYNA ISABEL OCHOA LANDÍN (D), Universidad Estatal de Sonora, Sonora, México

\section{Volumen 2, número 1}

Junio 2017

p. $1-24$

Este número se publicó el 30 de junio de 2017

Artículo recibido: 16 de febrero de 2017

Artículo Aprobado: 18 de mayo de 2017

ISSN: 2448-5942, doi: https://doi.org/10.36799/el.v2i1.43

\section{Cómo citar este artículo:}

Salado Rodríguez, L. I., Ramírez-Martinell, A., y Ochoa Landín, R. I. (2017). Hábitos de lectura y afinidad tecnológica de los estudiantes universitarios: estudio comparativo de cinco universidades de habla hispana. Estudios

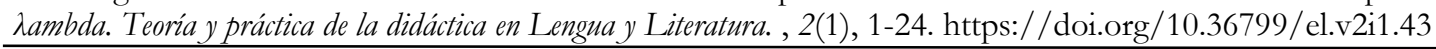

Derechos de autor: El autor o autores conservan en todo momento sus derechos morales y patrimoniales sobre la obra; la obra no se puede alterar, transformar o ampliar; siempre debe reconocerse la autoría del documento referido. Ninguna de las modalidades de los documentos publicados en Estudios Aambda. Teoría y práctica de la didáctica en lengua y literatura tienen fines comerciales de naturaleza alguna.

Los contenidos de este artículo están bajo una licencia de Creative Commons Atribución No Comercial- Sin Derivadas 4.0 Internacional (c) (1) () 


\title{
Hábitos de lectura y afinidad tecnológica de los estudiantes universitarios: estudio comparativo de cinco universidades de habla hispana ${ }^{1}$
}

\author{
Reading habits and technological affinity of university students: a comparative study of \\ five Spanish-speaking universities
}

\author{
LILIAN IVETTHE SALADO RODRÍGUEZ ${ }^{2}$ \\ ALBERTO RAMÍREZ-MARTINELL ${ }^{3}$ \\ REYNA ISABEL OCHOA LANDÍN ${ }^{4}$
}

\begin{abstract}
RESUMEN
Se realizó un estudio con grupos de estudiantes en cinco instituciones de educación superior nacionales y extranjeras, tres mexicanas (Universidad de Sonora, Universidad Veracruzana, Universidad Estatal de Sonora) una española (Universidad Complutense de Madrid) y una más argentina (Universidad Nacional de Jujuy), donde participó un total de 261 estudiantes de dos campos disciplinares: humanidades y económico-administrativo. La recolección de datos se llevó a cabo entre los meses de febrero a mayo del 2016, siendo la encuesta "La alfabetización informacional para la mejora del desarrollo académico de los estudiantes universitarios" el instrumento utilizado. En la encuesta se indagaron cuestiones de acceso y utilización de herramientas digitales así como de prácticas, usos y costumbres alrededor de la lectura con el fin de establecer una posible relación entre la afinidad tecnológica de los universitarios y sus hábitos de lectura. Así como también para conocer cuáles son los soportes y medios preferidos para leer y saber si aspectos como la ubicación geográfica, sexo o disciplina del estudiante influye en esta preferencia. De acuerdo con los resultados revisados, no fue posible establecer una relación directa y positiva entre las habilidades en el manejo de las TIC y los hábitos lectores de los estudiantes universitarios, independientemente de la disciplina académica, el país o el género. Sin embargo, se observó que aun cuando los estudiantes no tienen un nivel óptimo de habilidades tienden a leer más en medios digitales.
\end{abstract}

PALABRAS CLAVE: hábitos de lectura, estudiantes universitarios, afinidad tecnológica

\begin{abstract}
A study was conducted with group of students from five both national and foreign higher education institutions, three of them Mexican (Sonora University, Veracruz University and Sonora State University), one Spanish (Madrid Complutense University) and finally, one Argentinian (Jujuy National University). A total of 261 students from two different fields, humanities and economy and administration, participated in the mentioned study. The data collection was carried out between February and May on the year 2016 and the tool used was a survey called "The informational literacy for the academic improvement of university students".
\end{abstract}

\footnotetext{
${ }^{1}$ Este artículo es producto de la Red Temática Literacidad Digital en la Universidad (RED-LDU), reconocida y aprobada por CONACyT desde mayo del 2016, conformada por investigadores, académicos y estudiantes de diversas instituciones de educación superior nacionales y del extranjero.

${ }^{2}$ Doctora en Ciencias Sociales por el Colegio de Sonora. Profesora e investigadora de tiempo completo en la Universidad Estatal de Sonora. Líder del Cuerpo Académico "Innovación y Desarrollo Educativo", dictaminado en Consolidación por el PRODEP. Su principal línea de investigación es uso de tecnologías digitales en el contexto académico universitario. Correo electrónico: lilian.salado@ues.mx

${ }^{3}$ Doctor en Investigación Educativa por la Universidad de Lancaster, Inglaterra. Los temas de investigación que cultiva oscilan principalmente en tres áreas: tecnología educativa; diseño de estrategias y herramientas digitales educativas; y TIC para el desarrollo. Es Investigador de tiempo completo de la Universidad Veracruzana y tiene el reconocimiento de nivel 1 en el Sistema Nacional de Investigadores. Correo: albramirez@uv.mx

${ }^{4}$ Maestra en Administración por la Universidad de Sonora. Profesora investigadora de tiempo completo en la Universidad Estatal de Sonora. Miembro del cuerpo académico "Innovación y Desarrollo Educativo". Correo: reyna.ochoa@ues.mx
}

Artículo recibido: 16 de febrero de 2017

Artículo enviado a corrección: 24 de abril de 2017

Aprobado: 18 de mayo de 2017

Salado, Ramírezy Ochoa doi: https://doi.org/10.36799/el.v2i1.43 Número 2, Año 2017, ISSN: 2448-5942 
Matters of access and use of the digital tools were searched in the survey. Practices, uses and customs around reading, with the purpose of establishing a possible relationship between university students' technologic affinity and their reading habits, were considered in the research.

We also looked to know which are the supports and preferred means to read and to know if aspects such as the geographical location, sex or discipline of the student influences this preference. According to the revised results, it was not possible to establish a direct and positive relationship between ICT management skills and the reading habits of university students, regardless of academic discipline, country or gender. However, it was observed that even when students do not have an optimal level of skills they tend to read more in digital media.

KEYWORDS: Reading Habits, University Students, Technological Affinity.

\section{INTRODUCCIÓN}

Las tecnologías de información y comunicación (TIC) han irrumpido en todos los ámbitos y el contexto asociado a las prácticas de lectura no es la excepción. Los jóvenes universitarios -quienes son nuestros sujetos de estudio- actualmente tienen acceso a diferentes medios de información (repositorios de libros o sitios para la descarga de archivos pdf y epub) y soportes tecnológicos para la lectura (e-book readers, tabletas, laptops) donde se pueden encontrar materiales textos de temáticas, calidades y niveles de confiabilidad diversos. Sin embargo, no contamos con evidencia empírica sobre la relación de las TIC en los hábitos de lectura de los jóvenes -si es que existiera alguna-. Partiendo del supuesto de que el acceso a contenido digital se da principalmente a través de la lectura, en esta investigación nos hemos dado a la tarea de explorar la posible relación entre las prácticas cotidianas de uso de herramientas digitales con las prácticas y hábitos de lectura de los jóvenes universitarios, con el fin de estudiar las posibles tensiones que guardan estos dos aspectos que resultan fundamentales para el desempeño escolar y profesional de los universitarios en la actualidad.

Leer es una de las principales actividades para la adquisición de información independientemente de si se trata de una plataforma física o digital. En el contexto universitario, la revisión de textos para su futura discusión es clave, por lo que la investigación relacionada con las prácticas y habilidades y hábitos de lectura de los estudiantes se mantienen como temas centrales de estudio en la universidad y en otros niveles de enseñanza. Para el caso de educación básica por ejemplo, la Organización para la Cooperación y Desarrollo Económico (OCDE), incluyó en los reportes de los años 2000 y 2009, la lectura como área temática concreta del Programa Internacional para la Evaluación de los Alumnos (PISA) que se realiza cada 3 años a estudiantes de 15 y 16 años de los países miembros de la OCDE. De igual manera, la 
Organización de las Naciones Unidas para la Educación, la Ciencia y la Cultura (UNESCO), ha incluido la lectura como parte de sus indicadores la cultura, sólo por mencionar dos referentes mundiales que son usualmente citados con respecto a la evaluación del desempeño de los estudiantes.

En la educación universitaria, no existen indicadores de lectura nacionales o internacionales como los mencionados para educación básica. Empero los programas educativos dedican - en mayor o menor medida- una serie de experiencias educativas -regularmente al inicio de los programas de estudio- al desarrollo de habilidades lectoras, de aprendizaje, de escritura y de comunicación que son de carácter común para la formación general, en la totalidad de los programas educativos, sin que haya diferenciaciones en cuanto a la disciplina que se está cursando.

En la universidad -institución históricamente textocéntrica- la lectura de bibliografía propia de las asignaturas que comprenden el plan educativo es fundamental para conocer, analizar y apropiarse de las corrientes teóricas, la complejidad de los razonamientos y las bases empíricas que los apoyan (Carlino, 2005). Sin embargo, la preparación de los estudiantes en el tema es limitada, en parte porque se confía en que los estudiantes ya cuentan con esas habilidades que debieron haber adquirido en su trayectoria académica previo a los estudios profesionales y también debido a la tendencia, cada vez más generalizada en las universidades, de simplificar el currículo y reducir el tiempo de permanencia del estudiante donde las primeras materias que se “sacrifican”, por así decirlo, son de carácter general. Por ejemplo, en las cinco universidades en las que realizamos la investigación encontramos que la carga académica no supera a las tres asignaturas. En la Universidad Veracruzana hay dos asignaturas generales, una llamada Lectura y Redacción a través del Análisis del Mundo Contemporáneo y otra más sobre Habilidades del Pensamiento; en la Universidad Estatal de Sonora los estudiantes deben cursar tres asignaturas similares llamadas Fomento a la lectura, Comunicación Oral y Escrita, Aprendizaje y Gestión del Conocimiento; y en la Universidad de Sonora las asignaturas relativas al tema de lectura son dos: Comunicación Oral y Escrita y Aprendizaje y Gestión del Conocimiento. En las Universidades Española y Argentina, identificamos a los Seminarios de Lectura y el Taller de Lectura y Escritura, respectivamente como asignaturas para la procuración de la habilidad lectora de los estudiantes universitarios. 
La enseñanza de la lectura y escritura en el nivel universitario, según Carlino (2005), no se enfoca en la atención de las deficiencias de los estudiantes en cuanto a sus habilidades y hábitos lectores. A pesar de que el estudiante tiene al menos 12 años en el sistema educativo y una de las primeras habilidades que adquirió fue leer, la cultura académica de la lectura con la que los nuevos universitarios ingresan a la educación superior -señala la autora- depende de un gran número de factores y no resulta del todo clara. Por lo cual y ante la irrupción de nuevos factores, como las herramientas digitales y con ello las nuevas posibilidades de lectura (en cuanto a plataformas y tipos de contenido), se hace necesario seguirla escrudiñando.

\section{CONCEPCIÓN DE LA LECTURA}

La lectura, de acuerdo con Barthes (1994), se concibe como la decodificación de letras, palabras y significación de un texto. Ante esta perspectiva tan general, es necesario tener claro desde qué enfoque se concibe a la lectura puesto que ésta es una práctica que puede ser analizada desde varios puntos de vista: el intelectual (como un componente de capital cultural), el educativo (para la adquisición de conocimiento), como proceso comunicativo (para recibir y transmitir información), como práctica sociocultural, entre otros. De acuerdo con Cassany (2006), no existe una forma de leer que sea única ya que cada texto, disciplina y comunidad cuenta con formas específicas de leer y de interpretar dicha lectura. Siguiendo esta lógica, para el presente trabajo retomamos la actividad lectora como una práctica sociocultural. Esta perspectiva hace alusión a que, tanto las palabras como el conocimiento previo de quien hace la lectura, se originan en la sociedad. El discurso compartido por un autor contiene sus ideas, que a su vez fueron influenciadas por las ideas de otros textos, de otros autores y de sus propias experiencias (Cassany, 2006).

Además, este enfoque, según Cassany y Morales (2008), adopta una perspectiva más etnográfica e interdisciplinaria y destaca la relevancia de los factores contextuales: la comunidad de hablantes, la retórica empleada, la organización social, las identidades y los roles del autor y del lector.

El lector, señala Petit (1999), debe ir hacia la lectura, muchas veces ayudado, lo que se pretende en todas las universidades y lo cual, además, implica un esfuerzo o un impulso hacia la 
apertura puesto que hay que conocer y aceptar la cultura del otro - del profesor de lectura que por lo general no es del área de especialidad que estudian los jóvenes, de la misma cultura de los jóvenes que es difícilmente comprendida por los adultos, así como también de una nueva cultura digital que entra en la ecuación a partir de la creciente interacción de las personas con las TIC en todos los ámbitos-.

Los jóvenes universitarios que participan en esta intervención, estudian diferentes disciplinas y, por lo tanto, leen diferentes tipos de textos y de diversas formas, contando además con la influencia de la institución, a través de sus modelos educativos y las herramientas institucionales disponibles para la comunidad académica, sin dejar de lado la gran influencia que muchas veces representa la conducción en el aula de sus profesores en las diferentes materias que cursan; es por eso que nos interesa acercarnos a sus prácticas lectoras como una actividad sociocultural que finalmente repercute también en su trayectoria académica.

\section{LA LECTURA Y LOS MEDIOS DIGITALES}

La incursión permanente de las TIC en las escuelas debida a la cada vez mayor exposición a diferentes dispositivos así como la implementación de estrategias que las utilizan, como las plataformas educativas, ha conducido a una redefinición del desarrollo de la enseñanza frontal, simultánea y homogénea, tal como indica González (2015). Los estudiantes demandan ahora una atención más personalizada que muchas veces es difícil de ofrecer; en cambio, se puede optar por modelos educativos y estrategias de enseñanza aprendizaje más flexibles que no son posibles diseñar, ni llevar a cabo si no conocemos plenamente sus prácticas.

En cuanto a la lectura, González (2015) apunta que el principio natural ha cambiado, desde la manufactura hasta la usabilidad del lector frente a sus terminales. En este cambio las lecturas no han sido estructuradas para llevarse a cabo linealmente; el hipertexto brinda una serie de opciones que encaminan al lector hacia otros segmentos de información, videos, imágenes, blogs, sólo por mencionar algunos. En este contexto se vuelve indispensable que el lector cuente con capacidades no sólo propias de la lectura tradicional, sino también las que se requieren para la lectura digital, tales como: clasificación, gestión y selección de información además de identificación de fuentes confiables, entre las más indispensables, puesto que el libro impreso,

Salado, Ramirezy Ochoa doi: https://doi.org/10.36799/el.v2i1.43 Número 2, Año 2017, ISSN: 2448-5942 
como paradigma de transmisión de conocimiento ya está avalado mientras que en las fuentes electrónicas, una de las falencias más notables es la deslegitimación como instrumento de transferencia de conocimiento e información (Sepúlveda y Suárez, 2012).

Lo anterior no significa que no existan suficientes fuentes digitales plenamente confiables o que no haya mecanismos para identificarlas sino que lamentablemente el desconocimiento de ello y las prácticas incorrectas al respecto han contribuido a la disparidad en cuanto a la legitimidad de las fuentes tradicionales y las nuevas a las que se pueden acceder por medio de las herramientas digitales.

\section{MEDICIONES DE LA LECTURA DE LOS PAÍSES DONDE SE UBICAN LAS INSTITUCIONES DE LA INTERVENCIÓN}

De acuerdo con Petit (1999), la proporción de lectores asiduos entre los jóvenes ha disminuido en los últimos veinte años a pesar de existir actualmente una mayor escolarización, de manera generalizada en todos los países. La lectura, afirma esta autora, puede ayudar a los jóvenes a ser un poco más sujetos de su propia vida, y no solamente objetos de discursos represivos o paternalistas. A pesar de esto, en los indicadores de lectura, específicamente en los países donde se ubican los jóvenes de las instituciones de educación superior de México, España y Argentina, se puede ver que los jóvenes -que se ubican en este grupo aunque no necesariamente representan a los universitarios- están alejados de la lectura, tanto de libros como de otro tipo de textos que se incluyen en los instrumentos de medición. Martínez y Civelli (2011), confirman mediante la revisión de estudios como la Encuesta Nacional de Juventud (ENJ) y la Encuesta Nacional de Lectura (ENL) que en México la lectura no es común de manera general en la población mexicana pero es menos común entre los jóvenes. Al respecto, podemos ver en los resultados del 2015 de la encuesta nacional de lectura que realiza el Consejo Nacional para la Cultura y las Artes de México (CONACULTA) que la población lee menos de 3 libros al año. De los que si leen, el 21\% declaró que lo hace por ocio y $40 \%$ por recreación. Una buena parte de los encuestados con estudios universitarios declaró tener libros impresos (40\%) y digitales $(38 \%)$ en su casa.

Salado, Ramírezy Ochoa doi: https://doi.org/10.36799/el.v2i1.43 Número 2, Año 2017, ISSN: 2448-5942 
Por su parte, en el contexto Español, la Encuesta de Hábitos y Prácticas Culturales 20142015, ubica a la lectura como la segunda actividad más popular (62.2\%). La asistencia presencial a una biblioteca se estima, en términos anuales, en el $22.7 \%$ mientras que el acceso virtual se estimó en $7.9 \%$. Tanto en la asistencia a la biblioteca como en el acceso a ella por Internet, resultaron determinantes tres variables: la situación laboral, la edad y el nivel de estudios. Por edad, los mayores niveles de asistencia, por mucho, son los registrados por los estudiantes quienes declararon como los principales motivos para acudir a una biblioteca a la búsqueda de información, $27.4 \%$ y el préstamo de libros, 24.5\%. La encuesta española distingue dos tipos de lectura (aquella que se realiza motivada por la profesión o estudios de los lectores y la realizada por motivos de ocio o pasatiempo). En los resultados se pone de manifiesto hasta qué punto los motivos no profesionales son importantes motores de la lectura, situándose los lectores anuales por este motivo en el 56\% de los investigados, frente al 29\% de la población que lee por motivos vinculados a su profesión o estudios (Ministerio de Educación, Cultura y Deporte, 2015).

En Argentina, de acuerdo con Moreno, García y Sardi (2014), se reportaron varios hallazgos a partir de la aplicación de la segunda Encuesta Nacional de Hábitos de Lectura (ENHL), realizada en el 2011, entre los principales tenemos que los lectores, mismos que fueron definidos como quienes leen durante 15 minutos o más algún tipo de material, ascendían al 90\%, que la lectura digital creció de un $21 \%$ de personas que la practicaban a un $48 \%$, y, entre los menores de 25 años, redondea el 75\%, siendo una práctica que no sustituye la lectura en formatos tradicionales, sino que la complementa. En esta encuesta se destacó también que los jóvenes prefieren más la lectura mediante herramientas interactivas (redes sociales, chats, blogs) y menos en los formatos analógicos adaptados a la PC (correos electrónicos, diarios, etcétera).

De manera más general, podemos observar en los indicadores de la agencia NOP World Culture Score, lo relativo al índice de lectura construido mediante entrevistas con 30 mil personas mayores de 13 años realizadas entre diciembre de 2004 y febrero de 2005. De los datos del NOP World sobre consumo de medios - expresado en horas por semana- observamos que en México se dedicaba mucho más tiempo a la televisión (11.6 horas por semana) que a la lectura (5.5) y al uso de Internet con fines distintos a los académicos y laborales (6.3) -recordemos que estamos hablando de 2004 y 2005 e Internet en esas fechas era distinto al Internet que conocemos ahora-. Para el caso de Argentina el consumo de medios es similar -televisión 14 horas por semana, 
lectura 5.9 y medios digitales 8.9- solamente que en Argentina el uso y consumo de radio es mayor que en España, México y el resto de los países que se incluyeron en la encuesta con cerca de 21 horas por semana.

En España el consumo de televisión y de medios digitales es el mayor de los tres países con 15.9 y 11.5 horas por semana, mientras que el tiempo dedicado a la lectura es similar al de México y Argentina con 5.8 horas. En la Tabla 1 se muestra una comparación del consumo de Televisión, Radio, Lectura e Internet para los tres países que estamos contextualizando.

Tabla 1. Horas por semana dedicadas al consumo de medios: Caso de México, Argentina y España.

\begin{tabular}{|l|c|c|c|c|}
\hline & Televisión & Radio & Lectura & $\begin{array}{l}\text { Internet con } \\
\text { fines de ocio }\end{array}$ \\
\hline México & 11.6 & 11.1 & 5.5 & 6.3 \\
\hline Argentina & 14 & 20.8 & 5.9 & 8.9 \\
\hline España & 15.9 & 9.9 & 5.8 & 11.5 \\
\hline
\end{tabular}

Fuente: Índice de Lectura del NOP World. http://www.prnewswire.com/news-releases/nop-world-culturescoretm-index-examines-global-media-habits-uncovers-whos-tuning-in-logging-on-and-hitting-the-books$\underline{54693752 . h t m l}$

Ampliando la mirada, observamos que en el tema de Lectura es la India con 10.7 horas por semana el país que lee más; Corea con 3.1 el que lee menos y que podemos hablar de una media mundial de 6.08 horas por semana que nos sirve para ubicar tanto a México, Argentina y a España por debajo de ella. En lo relativo al uso de computadora e Internet con fines de ocio, era Taiwán - en 2004 y 2005- con 12.6 horas por semana el país con un mayor consumo y México el de menor consumo, ante una media global de 9.04. Ver Tabla 2.

Tabla 2. Horas por semana dedicas al consumo de medios: valores máximo, mínimo y medio.

\begin{tabular}{|c|c|c|c|c|}
\hline & Televisión & Radio & Lectura & $\begin{array}{l}\text { Internet con } \\
\text { fines de ocio }\end{array}$ \\
\hline Máximo & $\begin{array}{c}\text { Tailandia } \\
22.4\end{array}$ & $\begin{array}{c}\text { Argentina } \\
20.8\end{array}$ & $\begin{array}{c}\text { India } \\
10.7\end{array}$ & $\begin{array}{c}\text { Taiwán } \\
12.6\end{array}$ \\
\hline Mínimo & $\begin{array}{c}\text { México } \\
11.6\end{array}$ & $\begin{array}{c}\text { China } \\
2.1\end{array}$ & $\begin{array}{c}\text { Corea } \\
3.1 \\
\end{array}$ & $\begin{array}{c}\text { México } \\
6.3\end{array}$ \\
\hline Media & 15.73 & 9.04 & 6.08 & 9.04 \\
\hline
\end{tabular}

Fuente: Elaboración propia a partir de la información de NOP World http://www.prnewswire.com/newsreleases/nop-world-culture-scoretm-index-examines-global-media-habits-uncovers-whos-tuning-in-logging-onand-hitting-the-books-54693752.html 
De lo anterior podemos observar que los tres países se encuentran por debajo de la media global de lectura; pero no se puede observar una relación directa con su consumo de medios digitales, ya que México es el más bajo de los países, mientras Argentina y España se encuentran por arriba de la media (9.04) y no solamente eso, sino que España está solamente un punto porcentual por debajo del máximo.

En lo relativo a lo tecnológico, para medir y analizar el uso de las principales herramientas digitales disponibles para los universitarios, se utiliza el concepto de Afinidad Tecnológica, que fue definido en el proyecto de Brecha Digital en Educación Superior (Ramírez Martinell, Casillas y Ojeda, 2012) como al conjunto de disposiciones y valoraciones que los actores universitarios tienen sobre las TIC, operacionalizadas mediante cuatro dimensiones de análisis: Dominio de las TIC; Mundo actual y TIC; Internet como apoyo o distracción y Hábitos y conductas en la cultura digital (Casillas, Ramírez Martinell y Ortega, 2016).

En cuanto a la lectura, se construyó un índice el cual denominamos "Índice de Habitualidad Lectora", donde se conjuntaron aspectos relacionados con los hábitos de lectura de los estudiantes así como otros aspectos secundarios (como la posesión de libros) que consideramos influyen también en sus prácticas de lectura.

\section{METODOLOGÍA}

El presente estudio se llevó a cabo con grupos de estudiantes de diferentes programas educativos en las áreas disciplinares de humanidades y económico-administrativo de nivel licenciatura en tres universidades mexicanas -la Universidad Estatal de Sonora (UES), la Universidad de Sonora (UNISON) y la Universidad Veracruzana (UV)-; una universidad española -la Universidad Complutense de Madrid (UCM)-; y una universidad argentina -la Universidad Nacional de Jujuy (UNJ)-.

Para realizar la recolección de datos se utilizó una encuesta estandarizada que sirvió para recoger información escrita de una muestra de 261 estudiantes de ocho diferentes programas educativos de cinco universidades. El cuestionario denominado "La alfabetización informacional para la mejora del desarrollo académico de los estudiantes universitarios" -que puede ser consultado en https://docs.google.com/forms/d/1wmWbJ1Z2OzhhB3vFul2RYQEvtG14 GS6 
$\mathrm{dg} 5 \mathrm{HsPcCZb4} /$ viewform? $=0 \& w=1 \& u s p=$ mail form link, se les proporcionó a los estudiantes en formato electrónico mediante un sitio de Internet. Aunque dicho cuestionario contenía instrucciones descritas en el texto, al momento de aplicarse se dieron las mismas de manera oral por parte del aplicador con la finalidad de que los encuestados tuvieran claro el procedimiento para contestar el instrumento.

Una vez terminada la recepción de cuestionarios, las respuestas -que se fueron guardando automáticamente en una base de datos en Internet- se analizaron utilizando el programa SPSS (Statistical Package for the Social Sciences) versión 22 para obtener un análisis descriptivo que comprendió frecuencias y tablas cruzadas, de las respuestas obtenidas en los diferentes ítems del cuestionario, posteriormente se exportaron los datos a Microsoft Excel versión 2012 para la construcción de tablas y gráficos.

\section{POBLACIÓN}

La población con la que trabajamos tiene las siguientes características.

Género y Edad. El 71.3\% son mujeres. El 23\% tienen menos de 18 años; el 40\% tiene entre 19 y 20 años; el 18\% tienen entre 21 y 22 y el 20\% tiene más de 23 años.

Áreas académicas. El 64.5\% está inscritos en algún programa del área académica de humanidades y el 35.5\% en programas del área económico-administrativo.

País. El 22.9\% de los encuestados es de la universidad Argentina, el 21.37\% de la universidad Española y el $55.7 \%$ de las instituciones mexicanas (20\% de la UES, $18 \%$ de la UNISON y $18 \%$ de la UV).

En cuanto a su situación académica, el 80.8\% son alumnos regulares, lo cual implica que no tienen asignaturas o cursos reprobados, $11.5 \%$ son alumnos irregulares, que sí han reprobado algún curso y $7.7 \%$ cursa sus estudios en un formato libre en el cual no se le solicita llevar cierto número de créditos en un período de tiempo determinado. Al momento de realizar la intervención, $76.2 \%$ de los estudiantes cursaban su primer año en la universidad (en primer o segundo semestre) y el porcentaje restante se repartía entre los diferentes niveles, siendo el más numeroso el que correspondió al sexto semestre con $12.35 \%$ y al octavo semestre con un 5.7\%.

Salado, Ramirezy Ochoa doi: https://doi.org/10.36799/el.v2i1.43 Número 2, Año 2017, ISSN: 2448-5942 


\section{OPERACIONALIZACIÓN DE LAS VARIABLES}

Para explorar la posible relación entre los hábitos de lectura de los estudiantes con su afinidad tecnológica, se crearon dos índices mayores compuestos de variables ordinales y nominales a las que se les asignaron pesos dependiendo de su rol en el índice. A continuación se describe la operacionalización de las variables que componen tanto al índice de Habitualidad Lectora (iHL) como al índice de Afinidad Tecnológica (iAFI).

\section{ÍNDICE DE HABITUALIDAD LECTORA}

El índice de Habitualidad Lectora nos permite observar de manera global las percepciones que tienen los estudiantes sobre sus propios hábitos de lectura. Para su construcción se consideró de la encuesta los ítems relativos a sí tenían el hábito de la lectura, consideraciones de dicho hábito, la cantidad de libros que tenían en su casa, las horas que dedicaban por semana a lectura voluntaria, las horas que dedicaban a la semana a la lectura académica, la frecuencia con la que leían algunos tipos de libros y la capacidad para inferir información al revisar textos académicos. Ver Tabla 3.

Tabla 3. Construcción del índice de Habitualidad Lectora de los estudiantes.

\begin{tabular}{|c|c|c|}
\hline & Valores & $\begin{array}{c}\text { Puntajes máximo y } \\
\text { mínimo }\end{array}$ \\
\hline Hábito lectura & $\begin{array}{l}0-\mathrm{No} \\
1-\mathrm{Si}\end{array}$ & $\begin{array}{l}\text { Máximo: } 1 \\
\text { Mínimo: } 0\end{array}$ \\
\hline Causas & $\begin{array}{l}\text { - 1- Disfruto/Me gusta leer } \\
\text { libros o artículos por horas } \\
\text { - 1- Leo libros por lo menos } \\
\text { una vez a la semana } \\
\text { - 1- No me cuesta trabajo } \\
\text { leer libros } \\
\text { - } 0 \text { - Leo por necesidad y sólo } \\
\text { material de la escuela } \\
\text { - 0- Me aburre leer libros } \\
\text { - 0- Me quedo dormido } \\
\text { después de iniciar la lectura } \\
\text { de algún artículo o libro } \\
\text { - 0- Me cuesta trabajo } \\
\text { concentrarme }\end{array}$ & $\begin{array}{l}\text { Máximo: } 3 \\
\text { Mínimo: } 0\end{array}$ \\
\hline
\end{tabular}

Salado, Ramírez y Ochoa doi: https://doi.org/10.36799/el.v2i1.43 Número 2, Año 2017, ISSN: 2448-5942 


\begin{tabular}{|c|c|c|}
\hline Libros en tu casa & $\begin{array}{l}\text { - } 0 \text { - Ninguno } \\
\text { - } 1 \text { - De } 1 \text { a } 10 \\
\text { - 2- Entre } 11 \text { y } 24 \\
\text { - 3- Entre } 25 \text { y } 49 \\
\text { - 4- Entre } 50 \text { y } 100 \\
\end{array}$ & $\begin{array}{l}\text { Máximo: } 4 \\
\text { Mínimo: } 0\end{array}$ \\
\hline $\begin{array}{l}\text { Horas de lectura voluntaria a } \\
\text { la semana (en formato } \\
\text { impreso y digital) }\end{array}$ & $\begin{array}{l}0-0 \text { horas } \\
1-1-3 \text { horas } \\
2-4-7 \text { horas } \\
3-8 \text { a } 10 \text { horas } \\
4-\text { más de } 10 \text { horas }\end{array}$ & $\begin{array}{l}\text { Máximo: } 4 \\
\text { Mínimo: } 0\end{array}$ \\
\hline $\begin{array}{l}\text { Horas de lectura académica a } \\
\text { la semana (en formato } \\
\text { impreso y digital) }\end{array}$ & $\begin{array}{l}0-0 \text { horas } \\
1-1-3 \text { horas } \\
2-4-7 \text { horas } \\
3-8 \text { a } 10 \text { horas } \\
4-\text { más de } 10 \text { horas }\end{array}$ & $\begin{array}{l}\text { Máximo: } 4 \\
\text { Mínimo: } 0\end{array}$ \\
\hline $\begin{array}{l}\text { Libros NO académicos } \\
\text { leídos el año pasado }\end{array}$ & $\begin{array}{l}0 \text { - Ninguno } \\
1 \text { - De } 1 \text { a } 3 \\
2 \text { - De } 4 \text { a } 6 \\
3 \text { - De } 7 \text { a } 10 \\
4 \text { - Más de } 10\end{array}$ & $\begin{array}{l}\text { Máximo: } 4 \\
\text { Mínimo } 0\end{array}$ \\
\hline $\begin{array}{l}\text { Textos que comúnmente lees: } \\
\text { (Partes o capítulos de libros, } \\
\text { historietas, novelas o cuentos, } \\
\text { páginas o blogs, periódicos, } \\
\text { revistas, entre otros). }\end{array}$ & $\begin{array}{l}\text { 0- Nunca } \\
\text { 2- A veces } \\
\text { 4- Siempre }\end{array}$ & $\begin{array}{l}\text { Máximo: } 32(8 x 4) \\
\text { Mínimo: } 0\end{array}$ \\
\hline $\begin{array}{l}\text { Capacidad para inferir } \\
\text { información de un texto }\end{array}$ & $\begin{array}{l}0-\text { Nunca } \\
\text { 2- A veces } \\
\text { 4- Siempre }\end{array}$ & $\begin{array}{l}\text { Máximo: } 52(13 \times 4) \\
\text { Mínimo: } 0\end{array}$ \\
\hline $\mathrm{iHL}$ & & $\begin{array}{l}\text { Máximo: } 104 \\
\text { Mínimo: } 0\end{array}$ \\
\hline
\end{tabular}

Para analizar de iHL se hicieron las sumatorias de los resultados de cada uno de los estudiantes y la división - a partir de una lógica de cuartiles- mediante el establecimiento de tres cortes: iHL bajo, medio o alto.

\section{ÍNDICE DE AFINIDAD TECNOLÓGICA}

Para calcular el índice de Afinidad Tecnológica realizamos una suma de los valores de las cinco preguntas de la encuesta que se les hicieron a los estudiantes sobre el número de dispositivos digitales con los que contaban; el acceso y tiempo de conexión a Internet; las 
percepciones que tenían sobre su desempeño utilizando herramientas tecnológicas y su frecuencia de acceso y uso. La distribución de los valores y puntajes máximos y mínimos de los indicadores se muestra en la siguiente tabla.

Tabla 4. Construcción del índice de Afinidad Tecnológica de los estudiantes.

\begin{tabular}{|c|c|c|}
\hline & Valores & Puntajes máximo y mínimo \\
\hline Cantidad de dispositivos & $\begin{array}{l}0 \text {-sin dispositivos } \\
1-\text { al menos un dispositivo } \\
2-2 \text { dispositivos distintos } \\
3-3 \text { dispositivos distintos } \\
4-4 \text { dispositivos distintos }\end{array}$ & $\begin{array}{l}\text { Máximo: } 4 \\
\text { Mínimo: } 0\end{array}$ \\
\hline Acceso a contenido digital & $\begin{array}{l}\text { Cada opción distinta a no } \\
\text { accedo vale } 1 \\
\text { Puntaje máximo } 32\end{array}$ & $\begin{array}{l}\text { Máximo: } 32=(8 \times 4) \\
\text { Mínimo: } 0\end{array}$ \\
\hline $\begin{array}{l}\text { Tiempo de uso de Internet } \\
\text { (en horas) }\end{array}$ & $\begin{array}{l}\text { 0- No lo Utilizo } \\
\text { 1- } 1 \text { hora o menos } \\
\text { 2- } 2 \text { a } 4 \text { horas } \\
\text { 3- } 5 \text { a } 6 \text { horas } \\
\text { 4- Más de } 6 \text { horas. }\end{array}$ & $\begin{array}{l}\text { Máximo: } 20=(5 \times 4) \\
\text { Mínimo: } 0\end{array}$ \\
\hline Desempeño digital & $\begin{array}{l}0 \text { - Nulo } \\
1 \text { - Básico } \\
2-\text { Intermedio } \\
3 \text { - Avanzado } \\
\end{array}$ & $\begin{array}{l}\text { Máximo: } 33=(6 \times 3)+(5 \times 3) \\
\text { Mínimo: } 0\end{array}$ \\
\hline $\begin{array}{l}\text { Frecuencia de uso de } \\
\text { herramientas digitales con } \\
\text { fines de aprendizaje (chat, } \\
\text { correo electrónico, redes } \\
\text { sociales, plataformas } \\
\text { educativas, mensajes de } \\
\text { texto, videollamadas). }\end{array}$ & $\begin{array}{l}0-\text { Nunca } \\
2-\text { A veces } \\
4-\text { Siempre }\end{array}$ & $\begin{array}{l}\text { Máximo:24 = (6 x 4) } \\
\text { Mínimo: } 0\end{array}$ \\
\hline iAFI & & $\begin{array}{l}\text { Máximo: } 113 \\
\text { Mínimo: } 0\end{array}$ \\
\hline
\end{tabular}

Para analizar el iAFI se hicieron las sumatorias de los resultados de cada uno de los valores asociados a las respuestas que eligieron los estudiantes y de igual manera los dividimos a partir de una lógica de cuartiles en donde pudimos identificar un iAFI bajo, medio o alto. 


\section{RESULTADOS}

El análisis de los 261 estudiantes que contestaron la encuesta lo hemos organizado en tres partes. Primero hemos de presentar los conteos simples de ambos índices -Habitualidad Lectora y Afinidad tecnológica- con la finalidad de obtener un panorama global de la población en torno a estos dos temas. Posteriormente y debido a que este enfoque inicial no nos permite explicar una correlación entre los hábitos de lectura y la afinidad tecnológica de los estudiantes, hemos realizado el mismo análisis bajo tres miradas distintas; una para ver si el país de origen explica la correlación entre iAFI e iHL; y dos más para ver si es el género o la disciplina los que explican la correlación de índices en caso de que ésta existiera. Finalmente hemos realizado un análisis de las plataformas de lectura que utilizan los estudiantes, para poder observar si existen preferencias hacia los soportes y medios digitales. A continuación se presentan las tres secciones de análisis.

\section{MIRADA GENERAL Y CONTEOS SIMPLES}

En un rango de 0 a 1, observamos que el iHL promedio de los 261 estudiantes es de 0.54 con un máximo de 0.86 , un mínimo de 0.21 y una desviación estándar de 0.11 ; mientras que el promedio del iAFI de los estudiantes universitarios es de 0.44 - con un valor máximo de 0.70 , un mínimo de 0.12 y una desviación estándar de 0.9-.

La revisión global de los índices permite ver comportamientos generales, máximos y mínimos de la población, pero tratar de explicarlos, se requiere de una revisión específica bajo distintos lentes. A continuación presentamos un análisis de los datos bajo tres perspectivas: la geográfica, la disciplinaria y la de género.

\section{MIRADAS GEOGRÁFICA, DISCIPLINARIA Y DE GÉNERO}

La revisión de los datos considerando una mirada geográfica segmentada en tres rangos de valores -alto, medio y bajo- nos permite observar que ninguno de los países tienen valores en el rango "alto" y que más de la mitad de los encuestados tienen un índice bajo tanto en iAFI como en iHL. Ver Tabla 5.

Salado, Ramírezy Ochoa doi: https://doi.org/10.36799/el.v2i1.43 Número 2, Año 2017, ISSN: 2448-5942 
Tabla 5. Comparativo del iAFI -iHL por país.

\begin{tabular}{|c|c|c|c|c|c|c|}
\hline & iAFI & iHL & iAFI & iHL & iAFI & iHL \\
\hline & \multicolumn{2}{|c|}{ Вајо } & \multicolumn{2}{|c|}{ Medio } & \multicolumn{2}{|c|}{ Alto } \\
\hline México & $74 \%$ & $51 \%$ & $26 \%$ & $48 \%$ & $0 \%$ & $1 \%$ \\
\hline Argentina & $82 \%$ & $30 \%$ & $18 \%$ & $70 \%$ & $0 \%$ & $0 \%$ \\
\hline España & $77 \%$ & $25 \%$ & $23 \%$ & $75 \%$ & $0 \%$ & $0 \%$ \\
\hline
\end{tabular}

Al dividir a los informantes en dos grupos dependiendo de sus disciplinas académicas y buscar las posibles relaciones entre ambos índices podemos observar que al igual que en la mirada anterior, no hay valores en el rango “alto" y que los comportamientos entre los otros dos niveles (bajo y medio) pareciera no responder a ningún patrón tal y como se muestra en la Tabla 6.

Tabla 6. Comparativo del iAFI -iHL por disciplina.

\begin{tabular}{|l|r|r|r|r|r|r|}
\hline & \multicolumn{1}{|l|}{ iAFI } & iHL & iAFI & iHL & iAFI & iHL \\
\hline & \multicolumn{2}{|c|}{ Bajo } & \multicolumn{2}{|c|}{ Medio } & \multicolumn{2}{|c|}{ Alto } \\
\hline Humanidades & $\mathbf{8 3 \%}$ & $39 \%$ & $17 \%$ & $\mathbf{6 1} \%$ & $0 \%$ & $0 \%$ \\
\hline $\begin{array}{l}\text { Económico- } \\
\text { Administrativo }\end{array}$ & $\mathbf{6 3 \%}$ & $26 \%$ & $37 \%$ & $\mathbf{7 3} \%$ & $0 \%$ & $1 \%$ \\
\hline
\end{tabular}

Finalmente, al reagrupar a los 261 estudiantes de acuerdo a su género y explorar la posible relación entre ambos índices pudimos ver que la distribución de los datos no llega al nivel alto en ninguno de los índices y que para el caso de los varones más de la mitad se ubican en el segmento bajo como se muestra en la

Tabla 7.

Tabla 7. Comparativo del iAF - iHL por género.

\begin{tabular}{|l|l|l|r|r|r|r|}
\hline & \multicolumn{1}{|c|}{ iAFI } & \multicolumn{1}{l|}{ iHL } & iAFI & iHL & iAFI & iHL \\
\hline & \multicolumn{2}{|c|}{ Bajo } & \multicolumn{2}{c|}{ Medio } & \multicolumn{2}{c|}{ Alto } \\
\hline Femenino & $\mathbf{7 7 . 5 4 \%}$ & $36 \%$ & $22.46 \%$ & $\mathbf{6 4} \%$ & $0.00 \%$ & $0 \%$ \\
\hline Masculino & $\mathbf{7 3 . 3 3 \%}$ & $\mathbf{5 2} \%$ & $26.67 \%$ & $\mathbf{4 7 \%} \%$ & $0.00 \%$ & $1 \%$ \\
\hline
\end{tabular}

De lo anterior podemos concluir que con los datos que tenemos no es posible explicar la relación entre los dos índices ni de forma positiva ni negativa. Con esto no queremos decir que este resultado pueda ser generalizable o que esta intervención sea definitiva o errónea. Pero para el caso específico de los estudiantes de estas cinco universidades, la posible tensión entre el 
iAFI y el iHL no se explica ni al observar la cuestión geográfica - es decir que los estudiantes españoles, argentinos y mexicanos tienen un iAFI bajo y un iHL medio en su mayoría y que por tratarse de resultados similares su ubicación geográfica no explica la relación- ni al observar la disciplina académica -ya que de igual forma los estudiantes tienen un iAFI bajo y un iHL medioni el género de los estudiantes -ya que tanto hombres como mujeres tienen un iAFI bajo y un iHL medio-.

También debemos reconocer que las universidades que estudiamos son distintas, por un lado la universidad Española se ubica en la capital del país, es una universidad con recursos financieros y tecnológicos muy distintos a los de las otras universidades y podríamos pensar que el prestigio institucional convoca a estudiantes con perfiles distintos a los de aquellos que acuden a universidades no tan cosmopolitas como es el caso de la universidad argentina y las dos instituciones mexicanas que estudiamos.

El binomio iAFI bajo e iHL medio es recurrente bajo cualquiera de las tres segmentaciones de la población que realizamos, pero al igual que la manera genérica de observar los datos, esta exploración no nos permite explicar la relación o atribuirla a las cuestiones geográficas, disciplinarias o de género.

Finalmente la relación entre las cuestiones tecnológicas y de lectura de los estudiantes la procedimos a explorar con los modos preferidos para leer y el índice iAFI. El detalle de la exploración lo presentamos en el siguiente apartado.

\section{SOPORTES Y MEDIOS PREFERIDOS PARA LEER}

Para continuar con la exploración de la posible relación entre los hábitos de lectura y la afinidad tecnológica de los estudiantes universitarios, decidimos trascender el índice de Habitualidad Lectora -que consistía en los hábitos y razones para leer, el número de libros que tienen los estudiantes en casa, tiempo que le dedican a la lectura académica y de ocio- y enfocarnos en el soporte preferido para la lectura.

Sobre el soporte de lectura que prefieren los estudiantes, encontramos en la encuesta que aplicamos que los informantes de las cinco universidades tienen una clara preferencia por las

Salado, Ramírez y Ochoa doi: https://doi.org/10.36799/el.v2i1.43 Número 2, Año 2017, ISSN: 2448-5942 
páginas de Internet, al ser seguramente el medio más accesible, en la universidad de España tienden a despreciar los audiolibros, mientras que en México y Argentina, aunque en bajo porcentaje, se utilizan. Asimismo, pudimos observar que los estudiantes de la universidad argentina y la española hacen un uso indistinto de los libros electrónicos y los libros impresos (con pocas diferencias porcentuales); y que en México, la preferencia por el libro impreso sobre el electrónico tiene una diferencia mucho más significativa que en los demás países.

En la siguiente figura se pueden observar las proporciones de los medios preferidos para leer dependiendo la ubicación geográfica de los universitarios.

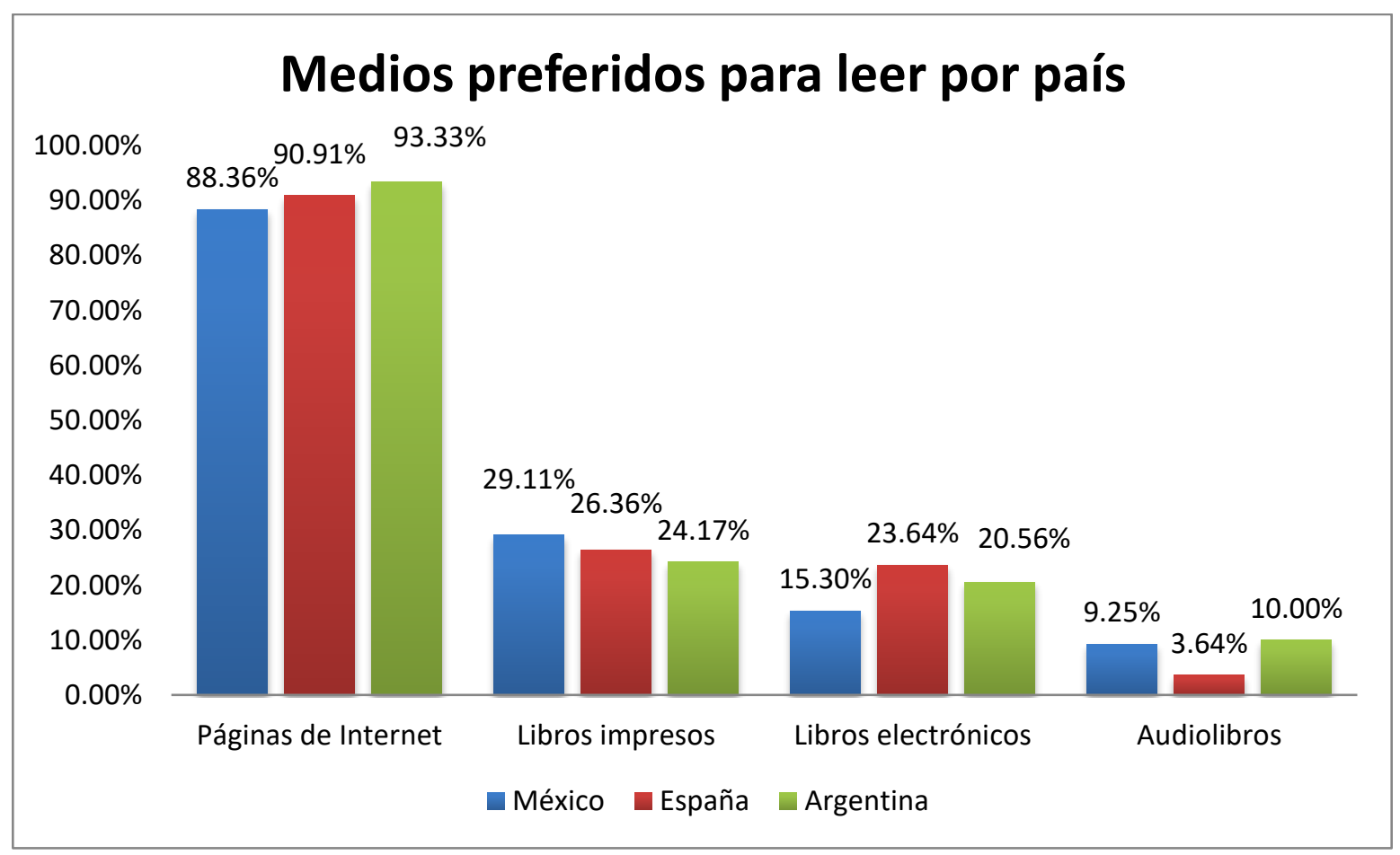

Figura 1. Medios preferidos para leer de los alumnos de acuerdo a la ubicación geográfica.

Con respecto a la disciplina de los estudiantes, tanto los de humanidades como los del área económico-administrativa, siguieron inclinándose claramente por las páginas de Internet; seguido - por los libros impresos y los libros electrónicos - con ligera diferencia entre ellos - y finalmente, los audiolibros. 


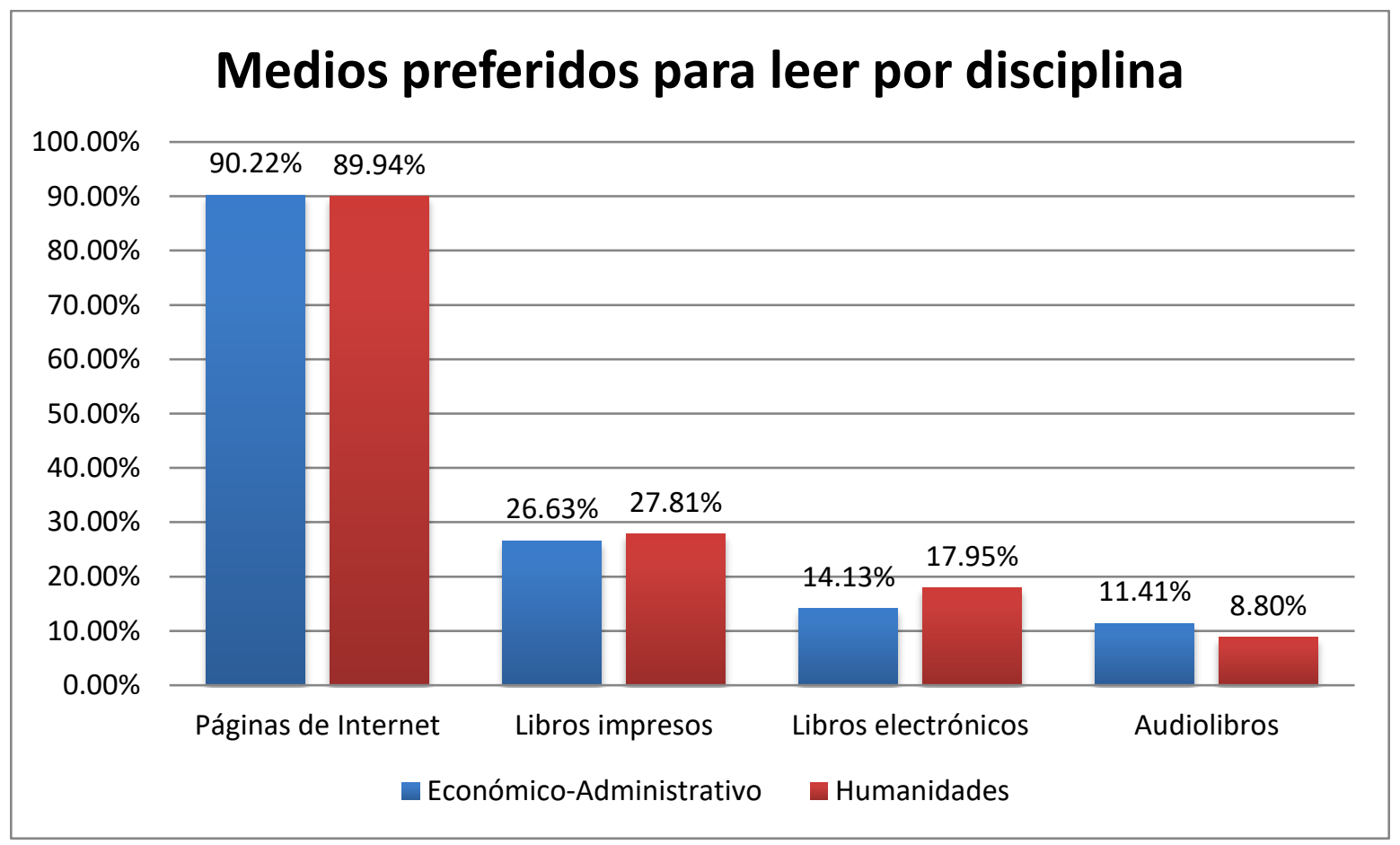

Figura 2. Medios preferidos para leer de los alumnos de acuerdo a la disciplina.

En cuanto al género, la Figura 3 nos muestra que, tanto hombres como mujeres, prefieren las páginas de Internet, seguido por el libro impreso aunque se puede observar una pequeña diferencia para el caso de los hombres, lo cual no sucede con los libros electrónicos donde prácticamente se muestran las mismas preferencias. 


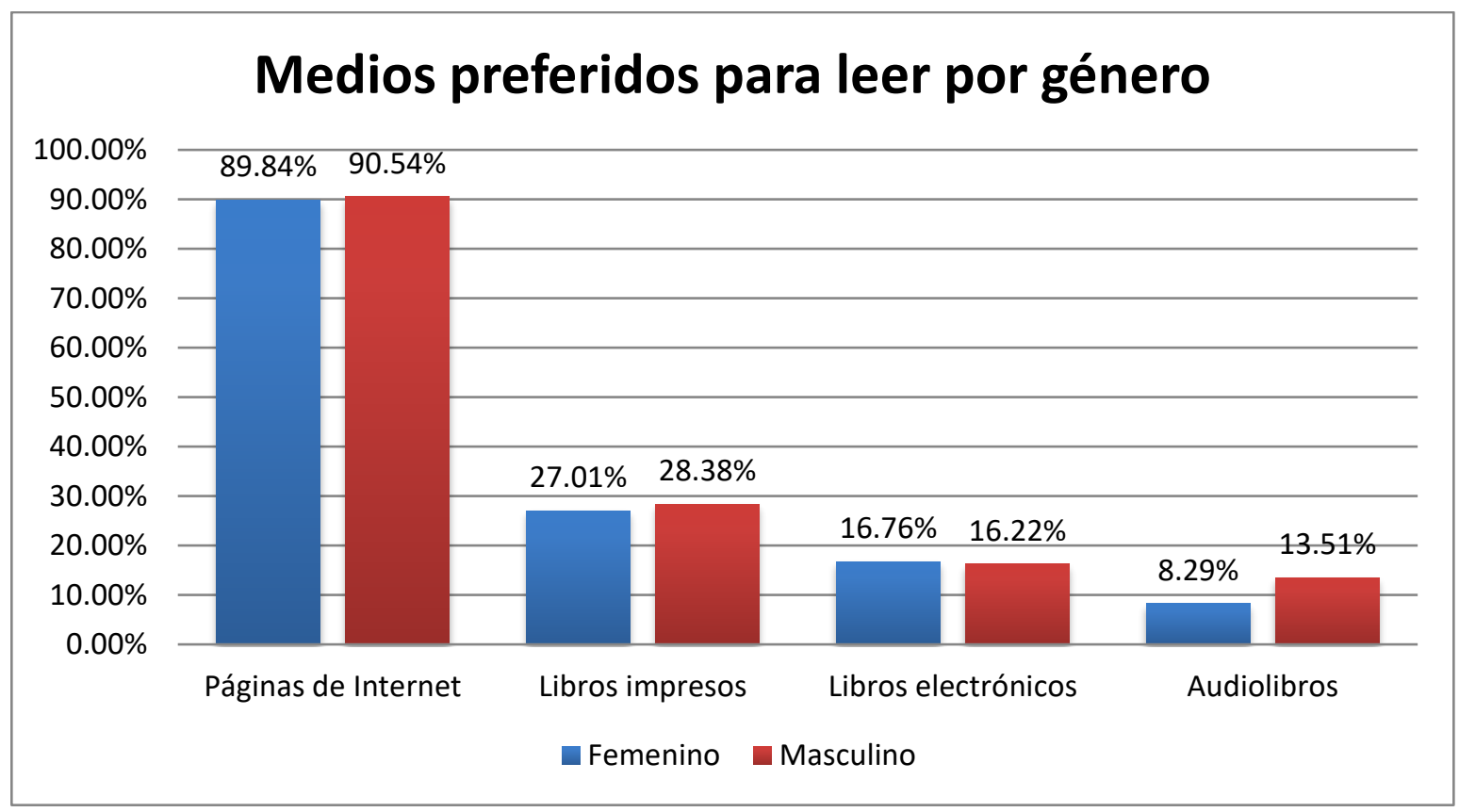

Figura 3. Medios preferidos para leer de los alumnos de acuerdo al género.

De manera más específica, en el ámbito académico, indagamos cuáles eran las fuentes a las que los estudiantes acudían para realizar sus tareas escolares. Sus respuestas, agrupadas por país, se concentran en la Figura 4, donde podemos observar nuevamente que es el contenido digital el que predomina, con las páginas de Internet y los libros electrónicos que se ubican por un margen no tan pronunciado detrás de los libros impresos. Es de llamar la atención que en España se supera por un amplio margen la utilización de revistas y periódicos electrónicos en comparación con lo observado en Argentina y México. 


\section{FUENTES UTILIZADAS PARA TAREAS}

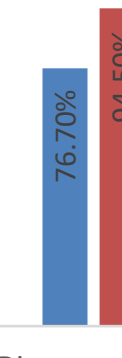

Diversas páginas de Internet

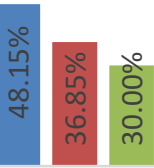

Libros impresos

Libros electrónicos

- ARGENTINA

- ESPAÑA

Figura 4. Fuentes utilizadas por los estudiantes para realizar sus tareas.

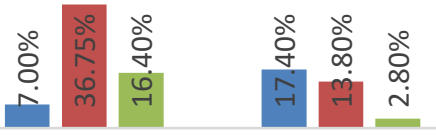

Revistas y periódicos electrónicos
Revistas y periódicos impresos

\section{MEXICO}

De igual manera, se indagó respecto a los tipos de texto que se consultan en Internet, siendo éste el medio preferido para los estudiantes para leer, en general, y de manera específica como un instrumento fundamental en su desempeño académico, nos pareció relevante conocer con mayor precisión el tipo de texto que consultan y leen en la red. Sus respuestas, de nuevo agrupadas por país, las podemos observar a detalle en la Figura 5.

\section{Tipos de textos digitales preferidos}

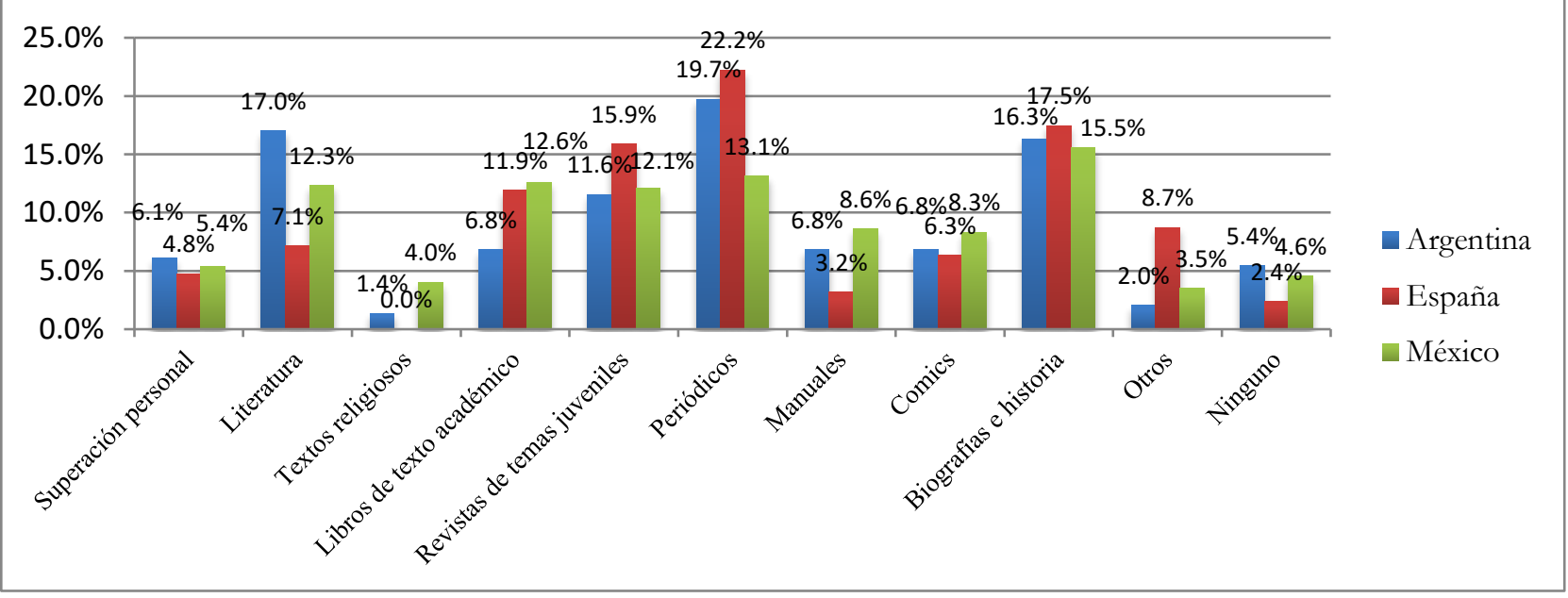

Figura 5. Tipos de textos digitales que leen los estudiantes.

Salado, Ramírezy Ochoa doi: https://doi.org/10.36799/el.v2i1.43 Número 2, Año 2017, ISSN: 2448-5942 
En México, los textos en formato digital que más consultan los estudiantes son las biografías o historia (15.5\%), seguido de periódicos (13.1\%) y textos académicos $(12.6 \%)$. Por su parte, los estudiantes españoles tienen entre sus preferencias los periódicos (22.2\%), seguido de biografías e historia (13.50\%) y textos académicos con 11.90\%. En Argentina, los textos digitales preferidos fueron los periódicos (19.70\%), seguido por literatura (17\%) y por último, biografías e historia $(16.30 \%)$.

Habría que resaltar que los libros de texto académico recibieron pocas menciones considerando que Internet es la fuente principal para la realización de tareas como los mismos estudiantes declararon.

\section{CONCLUSIONES}

Del análisis de los datos pudimos notar preferencias a fuentes de consulta digitales y a medios digitales para la lectura que resultaron ser contundentes para el caso de las páginas de Internet. Aun cuando inicialmente pensamos que podría existir una relación entre los hábitos de lectura y la afinidad tecnológica derivada principalmente del incremento del consumo de contenido de texto en las diversas pantallas de los estudiantes, los índices que construimos no fueron suficientes para explicar si su posible relación se debía al género, a la disciplina académica o a la región geográfica en la que vivían los estudiantes. Sin embargo, sí resultó muy claro que los jóvenes acuden a los medios digitales como su primera opción de lectura, tanto en el contexto académico como en el personal, Internet es su primer y principal fuente de consulta por su fácil disposición (aunque no en todos, en muchos de los casos), por la rapidez que representa, las posibilidades de acceso tanto en la escuela como en el hogar, incluso también porque los profesores disponen que lo hagan así, al menos en sus clases, poniendo a su disposición material digital y por medio de la institución a través de la implementación de plataformas educativas que se utilizan, principalmente, como repositorios para la diversa información que requieren las diferentes estrategias de enseñanza -aprendizaje (documentos, imágenes, videos, audios, etcétera).

Salado, Ramírezy Ochoa doi: https://doi.org/10.36799/el.v2i1.43 Número 2, Año 2017, ISSN: 2448-5942 
Si bien la construcción actual del índice iHL pareciera no relacionarse de forma alguna con el iAFI, al explorar con detenimiento a los soportes preferidos para la lectura sí pudimos identificar tres casos en los que se relacionan con la afinidad tecnológica de los estudiantes. Por un lado vimos que si los estudiantes tienen un iAFI alto preferirán leer en Internet y libros electrónicos, además de que podrán hacerlo con mayor destreza y con mejores posibilidades de consultar contenido de calidad; mientras que si se trata de estudiantes con un iAFI bajo tenderán a leer en soportes impresos o bien, corren el riesgo de no tener acceso a contenido confiable que es el grueso del contenido "rápido, fácil y accesible" con el que se topan los usuarios en la red y que dificultan la legitimación de este tipo de contenido; y finalmente también pudimos observar que si los estudiantes tienen un iHL bajo y un iAFI indistinto, tenderán a optar por audiolibros.

Como se señaló al inicio, retomamos la lectura desde una visión sociocultural que reconoce esta actividad como una construcción social y ha variado en los diferentes momentos de la historia, geografía y actividad de los seres humanos. En dicha visión Cassany (2006) ubica los diferentes géneros discursivos, es decir, no hay una sola forma de leer; cada texto, disciplina y comunidad tiene sus formas específicas de leerse e interpretarse. Aun así, de acuerdo a la indagación respecto a las fuentes preferidas por los estudiantes, se reveló que utilizan como fuente principal para sus tareas académicas textos en formato digital localizados en Internet. Estos textos constituyen la materia prima más importante cuando leen por motivos académicos. En este sentido, es importante mencionar como resultado de la investigación que los estudiantes cuentan con un iAFI medio o bajo por lo cual presentan deficiencias para seleccionar y aprovechar de manera óptima los distintos contenidos de Internet.

En las más recientes mediciones de la lectura se han incorporado los textos en formato digital, aún se está lejos de sistematizar una forma de conocer plenamente la interacción de los usuarios con este tipo de formatos disponibles a través de medios electrónicos. Por lo anterior, podemos expresar que ante la reconfiguración de las prácticas lectoras se deberán encontrar nuevas formas para medirlas e interpretarlas.

La exploración de la relación entre los medios digitales y su injerencia con la lectura de las personas sigue siendo una tarea pendiente que tendremos que seguir estudiando para poder conocer mejor a los estudiantes y poder generar políticas de adquisición de fuentes de información que les sean de real utilidad a estudiantes y profesores y no sólo como un accesorio

Salado, Ramírez y Ochoa doi: https://doi.org/10.36799/el.v2i1.43 Número 2, Año 2017, ISSN: 2448-5942 
para cumplir ciertos indicadores de calidad educativa, designación de espacios suficientes y adecuados para la lectura, y reestructuración de los planes de estudio en materia de formación de lectores.

\section{REFERENCIAS}

Barthes, Roland. El susurro del lenguaje más allá de la palabra y su escritura. España: Paidós, 2004.

Cassany, Daniel. Tras las líneas: sobre la lectura contemporánea. España: Anagrama, 2006.

Cassany, Daniel y Morales, Alberto. Leer y escribir en la universidad: Hacia la lectura y escritura crítica de géneros científicos. Revista Memoralia, 2008.

Carlino, Paula. Escribir, leer y aprender en la universidad. Argentina: Fondo de Cultura Económica, 2005.

Casillas, Miguel, Ramírez Martinell, Alberto, y Ortega, Juan. Afinidad tecnológica de los estudiantes universitarios. Revista Innovación Educativa del Instituto Politécnico Nacional, 16(70), 151-175, 2016.

Conaculta. Encuesta Nacional de hábitos, prácticas y consumo culturales. México: Conaculta, 2015.

González, Alejo. Las prácticas de lectura y escritura con TIC en el aula: apuntes para una investigación futura. Educación, Lenguaje y Sociedad, XII (12), 1-21, 2015.

Martínez, Pedro, Sivelli, Guillermo. Fomento a la lectura en los jóvenes mexicanos. En La lectura en el mundo de los jóvenes: ¿una actividad en riesgo?, compilado por Elsa Ramírez, 15-26. México: UNAM, 2011.

Ministerio de Educación, Cultura y Deporte. Encuesta de Hábitos y Prácticas Culturales 20142015. Síntesis de Resultados, 2015.

Moreno, Hilario, García, Santiago, Sardi, Valeria. Lectores, libros, lecturas: cambios en las prácticas y hábitos de lectura. Buenos Aires: Secretaría de Cultura de la Presidencia de la Nación, 2014. http://www.sinca.gob.ar/sic/publicaciones/libros/lectores-libros-lecturas.pdf

Salado, Ramírezy Ochoa doi: https://doi.org/10.36799/el.v2i1.43 Número 2, Año 2017, ISSN: 2448-5942 
NOP World. 07 de septiembre de 2016. http://www.prnewswire.com/news-releases/nopworld-culture-scoretm-index-examines-global-media-habits-uncovers-whos-tuning-inlogging-on-and-hitting-the-books-54693752.html

Petit, Michele. Nuevos acercamientos a los jóvenes y la lectura. Buenos Aires: Fondo de Cultura Económica, 1999.

Ramírez, Elsa (Coordinadora). La lectura en el mundo de los jóvenes: ¿una actividad en riesgo?. México: UNAM, 2011.

Ramírez Martinell, A. y Casillas, M. A. Los saberes digitales de los universitarios. En J. Micheli. Educación virtual y universidad, un modelo de evolución. Pp. 77-106. Serie Estudios Biblioteca de Ciencias Sociales y Humanidades. México: Universidad Autónoma Metropolitana, 2015.

Sepúlveda, Edwin y Suárez, Carlos. Libros electrónicos interactivos: nuevos lectores y nuevas terminales de lectura. Memorias del Encuentro Nacional de Investigación Hacia la Transformación de la Dinámica Investigativa, 2012. 